\section{Validade e fidedignidade de uma escala para avaliar autoeficácia para a atividade física do idoso}

\author{
Validity and reliability of a scale to assess self- \\ efficacy for physical activity in elderly
}

\begin{abstract}
Validez y la confianza en la escala para evaluar la autoeficacia de la actividad física de ancianos
\end{abstract}

Rossana Arruda Borges 1

Cassiano Ricardo Rech 1

Simone Teresinha Meurer 1

Tânia Rosane Bertoldo Benedetti 1

\footnotetext{
1 Universidade Federal

de Santa Catarina

Florianópolis, Brasil.

Correspondência

R. A. Borges

Universidade Federal de

Santa Catarina.

Campus Universitário Reitor João David Ferreira Lima,

Florianópolis, SC 80215-901,

Brasil.

rab_ametista@hotmail.com
}

\begin{abstract}
This study aimed to analyze the confirmatory factor validity and reliability of a self-efficacy scale for physical activity in a sample of $118 \mathrm{el}$ derly (78\% women) from 60 to 90 years of age. Mplus 6.1 was used to evaluate the confirmatory factor analysis. Reliability was tested by internal consistency and temporal stability. The original scale consisted of five items with dichotomous answers (yes/no), independently for walking and moderate and vigorous physical activity. The analysis excluded the item related to confidence in performing physical activities when on vacation. Two constructs were identified, called "self-efficacy for walking" and "self-efficacy for moderate and vigorous physical activity", with a factor load $\geq 0.50$. Internal consistency was adequate both for walking (>0.70) and moderate and vigorous physical activity ( $>0.80$ ), and temporal stability was adequate for all the items. In conclusion, the self-efficacy scale for physical activity showed adequate validity, reliability, and internal consistency for evaluating this construct in elderly Brazilians.
\end{abstract}

Self Efficacy; Validation Studies; Motor Activity; Aged

\section{Resumen}

Este estudo teve como objetivo analisar a validade fatorial confirmatória e a fidedignidade de uma escala de medida da autoeficácia para atividade física em idosos. Participaram 118 idosos (78\% mulheres), com idade entre 60 a 90 anos. Para avaliar a análise fatorial confirmatória utilizou-se o programa Mplus 6.1. A fidedignidade foi testada pela consistência interna e estabilidade temporal. A escala original foi composta por cinco itens com resposta dicotômica (não/sim), de maneira independente para caminhada e atividade física moderada e vigorosa. O item relacionado à confiança em realizar atividade física quando o mesmo está de férias foi excluído. Foram identificados dois construtos denominados "autoeficácia para caminhada" $e$ "autoeficácia para atividade física moderada e vigorosa", com carga fatorial $\geq 0,50$. Houve adequados valores de consistência interna, tanto para caminhada $(>0,70)$ quanto para atividade física moderada e vigorosa $(>0,80)$ e de estabilidade temporal para todos os itens. Conclui-se que a escala de autoeficácia para atividade física apresenta validade, consistência interna e fidedignidade adequada para avaliar esse construto em idosos brasileiros.

Autoeficácia; Estudos de Validação; Atividade Motora; Idoso 


\section{Introdução}

A percepção de autoeficácia reflete a crença na capacidade de realizar com sucesso uma atividade/comportamento ${ }^{1}$. Essa percepção pode influenciar as atividades realizadas, o tempo investido e a persistência em manter a mesma, especialmente quando o indivíduo se depara com barreiras para este comportamento 2 . A autoeficácia é um construto chave da teoria sociocognitiva e está associada com comportamentos positivos relacionados à saúde, dentre eles a atividade física 3,4 . Em idosos, observa-se um importante papel da autoeficácia para adoção da atividade física 2,5.

Elevar a autoeficácia para atividade física, especialmente em idosos, pode representar uma importante estratégia para a promoção da atividade física 2,4,6. Evidências confirmam que autoeficácia para atividade física é um importante mediador da efetividade para os programas de atividade física 2,7,8. Idosos com maior autoeficácia para atividade física podem aumentar a expectativa de sucesso diante de um comportamento, como manter a rotina de atividade física, mesmo diante de obstáculos que podem dificultar a adesão e manutenção nesta atividade 7. Existem poucos instrumentos válidos para avaliar a autoeficácia para atividade física no Brasil. Escalas para avaliar a autoeficácia podem ser gerais, ou seja, podem mensurar a confiança geral do indivíduo ou, estar relacionado com comportamentos específicos, como atividade física 9 .

Um estudo pioneiro, em 1988, propôs uma escala de 18 itens para avaliar a autoeficácia para atividade física 10. Essa escala, com opção de resposta de cinco pontos indica o nível de confiança para realizar atividade física em situações adversas. Outro estudo desenvolveu uma escala de dez itens, com respostas que variam de 0 (nada confiante) a 100 (muito confiante) 9 . Esta escala foi traduzida e adaptada para a população brasileira 11, porém, incluiu um número limitado de idosos, o que torna sua aplicabilidade nesta população desconhecida. Além disso, a escala avalia a percepção geral de autoeficácia para o exercício físico, não considerando diferença na percepção em relação à intensidade e o tipo de atividade física. Acredita-se que idosos podem apresentar diferença na autoeficácia para atividade física dependendo do tipo e da intensidade da mesma. Um idoso pode apresentar elevada confiança em caminhar, mas não reportar a mesma confiança para realizar atividade vigorosa.

Neste sentido, uma escala composta por cinco itens foi validada em adultos brasileiros e considerou dois fatores (autoeficácia para caminhada e autoeficácia para atividade moderada e vigorosa). Essa escala foi adaptada de um instrumento internacional e apresentou propriedades psicométricas adequadas em adultos 12 . Além disso, esse instrumento teve sua escala de resposta adaptada para resposta dicotômica, que, apesar de apresentarem menor variabilidade, podem ser de melhor compreensão na população de idosos. Acredita-se que a validação de uma escala para medir a autoeficácia para atividade física em idosos possa contribuir com novos achados sobre essa relação na população brasileira, pois, até o momento, não encontramos estudos demonstrando essa associação em amostras nacionais.

Deste modo, este estudo tem como objetivo analisar a validade fatorial confirmatória e a fidedignidade de uma escala de medida da autoeficácia para atividade física em uma amostra de idosos brasileiros.

\section{Métodos}

Foram analisados dados do baseline da intervenção em mudança de comportamento denominado VAMOS: Vida Ativa Melhorando à Saúde, desenvolvido em Centros de Saúde de Florianópolis, Santa Catarina, Brasil, em 2012. Foram avaliados 118 idosos (78\% mulheres), entre $60 \mathrm{e}$ 90 anos de idade. Após um período entre quatro e sete dias a escala de autoeficácia para atividade física foi reaplicada em 39 destes idosos $(82,1 \%$ mulheres) com objetivo de verificar a fidedignidade da escala. Idosos com debilidade física e/ ou mental grave não foram elegíveis no baseline. Os procedimentos foram aprovados no Comitê de Ética em Pesquisa da Universidade Federal de Santa Catarina (protocolo no 2.387/2012).

A escala de autoeficácia para atividade física, analisada neste estudo, é composta por cinco itens com repostas dicotômicas ("não" versus "sim”) e representa a crença na confiança em realizar atividade física mesmo quando existem barreiras para essa prática, como por exemplo, falta de tempo, frio, cansaço, etc. Originalmente essa escala apresentava cinco pontos de reposta ("nada confiante" até "muito confiante"). Entretanto, estudo de validação para a língua portuguesa propôs o uso de uma escala de respostas dicotômicas, no sentido de facilitar a compreensão para entrevistados com baixa escolaridade 9 e tornar facilitada a coleta de informações em estudos epidemiológicos. Além disso, considerando que a autoeficácia é específica para cada comportamento, utilizou-se a escala de acordo com diferentes intensidades de atividade física (caminhada e atividade física de intensidade moderada e vigorosa) 12 . O escore geral da escala 
varia de "zero" a "cinco" pontos, sendo que escores mais elevados refletem maior autoeficácia para atividade física. A escala original apresentou validade e consistência interna em adultos brasileiros demonstrando características psicométricas adequadas 12 .

As variáveis sexo (masculino/feminino), idade (anos), escolaridade, percepção de saúde e status de peso também foram avaliadas. A escolaridade foi agrupada de acordo com a última série escolar cursada (analfabeto/Fundamental incompleto; Fundamental completo/Médio incompleto; Médio completo; Superior completo). A percepção de saúde foi verificada com a questão “O(a) Sr(a). diria que sua saúde é...”, como opção de resposta uma escala de cinco pontos (muito ruim, ruim, regular, boa e muito boa). As opções "boa" e "muito boa" foram agrupadas em "percepção de saúde positiva", já as opções "regular", "ruim" e "muito ruim" foram agrupadas em "percepção de saúde negativa". Para classificação do status de peso utilizou-se o cálculo do índice de massa corporal (IMC) 13. Para coleta de dados a escala foi aplicada por indivíduos treinados em local reservado e adequado para aplicação.

As características dos participantes são apresentadas pela frequência absoluta e relativa. Os testes de McNemar e Friedman foram empregados para comparar as características da amostra de validade e fidedignidade. A validade fatorial foi avaliada por meio da análise fatorial confirmatória do programa Mplus 6.1 (Muthén \& Muthén, Los Angeles, Estados Unidos). Devido à utilização de escalas de respostas dicotômicas para os itens da escala optou-se por utilizar um estimador WLS (método de mínimos quadrados ponderados), que considera para efeito de estimativa as matrizes de covariância para correlação tetracóricas, ou seja, ajustadas para escalas dicotômicas. Este ajuste é importante, pois o padrão (default) do programa Mplus 6.1 realiza estimativas por meio do método da máxima verossimilhança, que requer distribuição normal O que não é plausível em dados categóricos 14 . Para tanto, consideraram-se como critérios de validade a carga fatorial do item $\geq 0,50$ e índices de ajuste do modelo aceitáveis (Comparative Fit Index - CFI $\geq 0$ 0,90; Tucker-Lewis Index - TFI $\geq$ 0,90; Root Mean Square Error of Aproximation RMSEA $\leq 0,08) 14$. A fidedignidade foi testada pela consistência interna ( $\alpha$ de Cronbach e coeficiente de kappa) e estabilidade temporal (coeficiente de correlação intraclasse), sendo a estabilidade temporal pelo método teste-reteste. Valores de $\alpha$ e coeficiente de correlação intraclasse $(\mathrm{CCI}) \geq$ 0,70 foram considerados como adequados 15 .

\section{Resultados}

As características da amostra estão apresentadas na Tabela 1. No geral, houve maior proporção de mulheres (78\%), com a faixa etária entre 60 e 69 anos $(52,6 \%)$, grau de escolaridade analfabeto/ Fundamental incompleto (61,8\%) e com excesso de peso (75,5\%). A percepção de saúde positiva foi relatada por $46,6 \%$ da amostra. Não houve diferença estatística significante entre as amostra de validade e reprodutibilidade.

Entre os dez itens testados, dois itens (AE4 e AE9), referentes à confiança em realizar a caminhada e atividade física moderada e vigorosa quando os idosos estiverem de férias, foram excluídos. A aplicação prática identificou que esses itens não têm significância para a população idosa. Assim, a escala final foi composta por oito itens, sendo quatro relacionados com a caminhada e quatro com a atividade física moderada e vigorosa (Figura 1).

A análise fatorial confirmatória testou dois fatores relacionados, rotulados de "autoeficácia para caminhada" e "autoeficácia para atividade física moderada e vigorosa" $(\mathrm{r}=0,66)$. Todos os itens apresentaram carga fatorial $\geq 0,50$ e os índices de ajuste do modelo foram considerados adequados $\left(\chi^{2}=24,647 ; \mathrm{p}=0,1029\right.$; $\mathrm{CFI}=0,989$; TFI = 0,982; RMSEA = 0,062; IC90\%: 0,001-0,112).

A análise da consistência interna ( $\alpha$ de Cronbach) demonstrou valor superior a 0,70 para caminhada e 0,80 para atividade física moderada e vigorosa. Os resultados das análises de estabilidade temporal apresentados na Tabela 2, mostram elevada concordância $(\% \mathrm{C})$ para todos os itens da escala, tanto para caminhada (\%C: $82,1 \%$ a $92,3 \%$ ) quanto atividade física moderada e vigorosa (\%C: $87,2 \%$ a 94,9\%). Ao considerar os escores totais de cada escala, os valores de CCI foram 0,78 para caminhada (IC95\%: 0,61-0,87) e 0,86 para atividade física moderada e vigorosa (IC95\%: 0,75-0,93).

\section{Discussão}

Os resultados demonstram que a escala de autoeficácia para atividade física em idosos possui validade confirmatória e fidedignidade satisfatória. Foram necessárias adaptações em relação ao instrumento original. Os itens AE4 e AE9 relacionados à confiança em realizar atividade física mesmo quando o idoso está de férias foram excluídos. Esta exclusão foi baseada na aplicação dos itens originais da escala, onde maior parte dos idosos relatou não trabalhar. Assim, optouse pela exclusão destes itens. Essa exclusão não prejudicou a validade estrutural da escala. 
Tabela 1

Características sociodemográficas, percepção de saúde e peso corporal dos idosos ( $N=118)$. Florianópolis, Santa Catarina Brasil, 2013

\begin{tabular}{|c|c|c|c|c|c|c|}
\hline \multirow[t]{2}{*}{ Variáveis } & \multicolumn{2}{|c|}{ Validação ( $n=118)$} & \multicolumn{2}{|c|}{ Fidedignidade $(n=39)$} & \multicolumn{2}{|c|}{ Estatística } \\
\hline & $\mathrm{n}$ & $\%$ & $\mathbf{n}$ & $\%$ & $\chi^{2} / \mathrm{F}$ & $\begin{array}{l}\text { Valor } \\
\text { de p }\end{array}$ \\
\hline Sexo * & & & & & 0,29 & 0,59 \\
\hline Mulheres & 92 & 78,0 & 32 & 82,1 & & \\
\hline Homens & 26 & 22,0 & 7 & 17,9 & & \\
\hline Faixa etária (anos) ** & & & & & 0,680 & 0,409 \\
\hline $60-64$ & 31 & 26,3 & 11 & 28,2 & & \\
\hline $65-69$ & 31 & 26,3 & 12 & 30,8 & & \\
\hline $70-74$ & 26 & 22,0 & 5 & 12,8 & & \\
\hline $75-79$ & 17 & 14,4 & 7 & 17,9 & & \\
\hline$\geq 80$ & 12 & 10,2 & 4 & 10,3 & & \\
\hline Escolaridade ** & & & & & 0,549 & 0,421 \\
\hline $\begin{array}{l}\text { Analfabeto/Fundamental } \\
\text { incompleto }\end{array}$ & 73 & 61,8 & 22 & 54,4 & & \\
\hline $\begin{array}{l}\text { Fundamental completo/ } \\
\text { Médio incompleto }\end{array}$ & 17 & 14,4 & 5 & 12,8 & & \\
\hline Médio completo & 15 & 12,7 & 6 & 15,4 & & \\
\hline Superior completo & 13 & 11,0 & 6 & 15,4 & & \\
\hline Percepção de saúde * & & & & & 3,84 & 0,789 \\
\hline Positiva & 55 & 46,6 & 20 & 51,3 & & \\
\hline Negativa & 62 & 52,5 & 19 & 48,7 & & \\
\hline Status de peso corporal ** & & & & & 0,874 & 0,213 \\
\hline Eutrófico *** & 24 & 20,3 & 8 & 20,5 & & \\
\hline Sobrepeso \# & 52 & 44,1 & 17 & 43,6 & & \\
\hline Obeso \#\# & 37 & 31,4 & 13 & 33,3 & & \\
\hline
\end{tabular}

* Teste de McNemar;

** Teste Friedman;

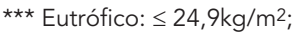

\# Sobrepeso: $25-29,9 \mathrm{~kg} / \mathrm{m}^{2}$;

\#\# Obeso: $\geq 30 \mathrm{~kg} / \mathrm{m}^{2}$.

A análise fatorial confirmatória testou dois fatores que foram denominados "autoeficácia para caminhada" e "autoeficácia para atividade física moderada e vigorosa” (Figura 1). A estrutura exploratória em dois fatores foi atribuída às diferentes habilidades exigidas em cada intensidade e tipo de atividade física, além da distinta percepção dos idosos em relação às suas capacidades para os dois níveis de exigência. Estes resultados reforçam a especificidade da autoeficácia ao comportamento em análise 9,12. A autoeficácia pode ser construída a partir de experiências vicárias, ou seja, a presença de idosos em caminhadas é mais comum do que em atividade física moderada e vigorosa, o que pode influenciar a forma com que estes idosos demonstram sua confiança para estas diferentes intensidades de atividade física 1,16 .

Os valores de $\alpha$ de Cronbach foram superiores a 0,70 (caminhada) e 0,80 (atividade física moderada e vigorosa), indicando adequada consistência interna da escala ${ }^{15}$. Ao excluir qualquer um dos itens que compõem os fatores, o valor de $\alpha$ diminuiu, confirmando que todos os itens contribuem de forma importante para a construção dos fatores. Estes valores são semelhantes aos relatados em adultos brasileiros 12 que demonstraram valores de $\alpha$ de Cronbach de 0,83 (caminhada) e 0,90 (atividade física moderada e vigorosa) e em outros estudos internacionais 6,17,18. Um estudo realizado com adultos e idosos brasileiros apontou um $\alpha=0,9011$. O valor mais 
Figura 1

Análise fatorial confirmatória da escala de autoeficácia para atividade física em idosos.

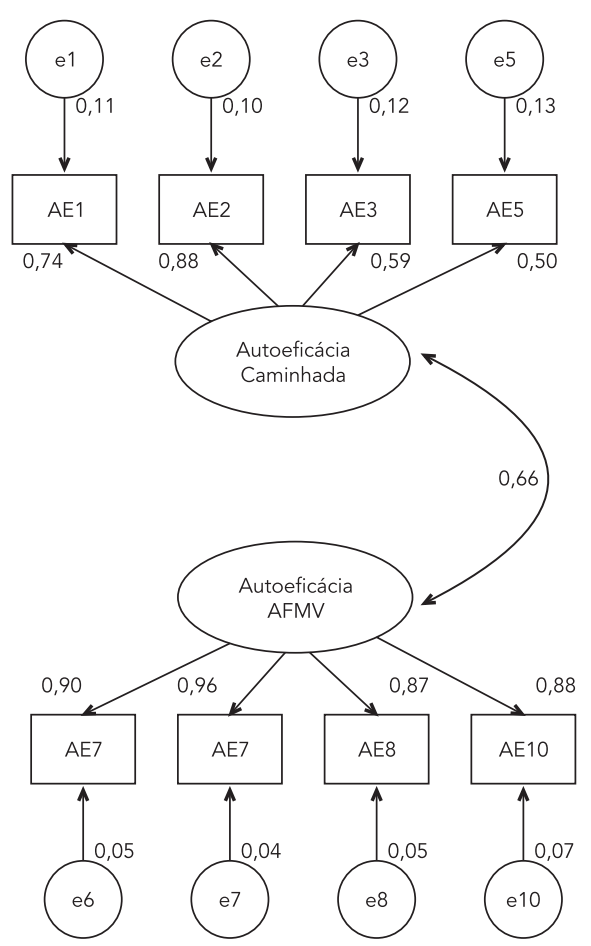

AFMV: atividade física moderada e vigorosa.

Nota: $N=118 ;$ d.f. $=56$; Estimador $=W L S M V ; \chi^{2}=24,647 ; p=0,1029 ; \mathrm{CFI}=0,989 ; \mathrm{TFI}=0,982 ; \mathrm{RMSEA}=0,062 ; \mathrm{IC} 90 \%$ :

$0,001-0,112$.

Tabela 2

Fidedignidade da escala de autoeficácia para a atividade física em idosos. Florianópolis, Santa Catarina, Brasil, 2013.

\begin{tabular}{|c|c|c|c|c|c|c|}
\hline \multirow[t]{2}{*}{ Item } & \multirow[t]{2}{*}{ Descrição do item } & \multicolumn{3}{|c|}{ Reprodutibilidade } & \multicolumn{2}{|c|}{ Consistência interna } \\
\hline & & $\% \mathrm{C}$ & Kappa * & $\mathrm{CCl}(\mathrm{IC} 95 \%)$ & $\begin{array}{l}\alpha \text { do fator se item } \\
\text { for deletado }\end{array}$ & $\alpha$ do fator \\
\hline \multicolumn{7}{|c|}{ Você se sente confiante em realizar caminhada mesmo.... } \\
\hline AE1 & ...quando você está cansado? & 87,2 & 0,75 & \multirow{4}{*}{$0,78 *(0,61-0,87)$} & 0,68 & 0,70 \\
\hline AE2 & ...quando você está de mau humor? & 92,3 & 0,79 & & 0,63 & \\
\hline AE3 & ...quando você está sem tempo? & 82,1 & 0,64 & & 0,67 & \\
\hline AE5 & ...quando está muito frio? & 84,6 & 0,64 & & 0,68 & \\
\hline \multicolumn{7}{|c|}{ Você se sente confiante em realizar atividade física moderada } \\
\hline \multicolumn{7}{|c|}{ e vigorosa mesmo... } \\
\hline AE6 & ...quando você está cansado? & 84,6 & 0,62 & \multirow{4}{*}{$0,86 *(0,75-0,93)$} & 0,81 & 0,84 \\
\hline AE7 & ...quando você está de mau humor? & 94,9 & 0,83 & & 0,77 & \\
\hline AE8 & ...quando você está sem tempo? & 94,9 & 0,88 & & 0,80 & \\
\hline AE10 & ...quando está muito frio? & 87,2 & 0,68 & & 0,82 & \\
\hline
\end{tabular}

\%C: percentual de concordância; CCl: coeficiente de correlação intraclasse; IC95\%: intervalo de $95 \%$ de confiança.

* $p<0,005$ para todos os itens. 
elevado em adultos é esperado, pois idosos possuem maior dificuldade em respostas subjetivas que incluem o julgamento de capacidades e habilidades físicas 16,19. Em relação à reprodutibilidade, observou-se elevada concordância entre todos os itens da escala (concordância relativa > $80 \%$ ) no teste e reteste (Tabela 2). O coeficiente de correlação intraclasse também foi significativo, tanto na caminhada (CCI $=0,78 ; \mathrm{p}<0,001$ ) quanto na atividade física moderada e vigorosa $(\mathrm{CCI}=0,86 ; \mathrm{p}<0,001)$. Estes resultados confirmam a estabilidade temporal da escala de autoeficácia para atividade física em idosos, sendo similar a resultados obtidos em outras amostras de idosos 6,17.

A autoeficácia para atividade física é relatada como importante determinante da atividade física em idosos 16,19,20. Neste sentido, acreditase que a validade desta escala contribuirá com os pesquisadores da área de saúde coletiva na elaboração de hipóteses de estudos relacionados a essa temática. Evidências apontam que um aumento na autoeficácia para caminhada pode estimular que indivíduos adotem esse comportamento em programas comunitários 20 . Este fato seria importante, pois a caminhada representa um dos mais prevalentes tipos de atividade física entre adultos e idosos 21 . Além disso, uma série de estratégias podem potencializar a autoeficácia para atividade física em idosos, como por exemplo, o encorajamento verbal e a aprendizagem vicária. Espera-se que com um instrumento que possibilite avaliar a autoeficácia para atividade física, nesta população, estas estratégias sejam implementadas em maior escala.

Algumas limitações necessitam ser consideradas para a extrapolação dos resultados encontrados. A amostra não incluiu idosos institucionalizados, com algum problema físico ou mental e houve uma predominância de mulheres tanto na amostra de validação (78\%) quanto de fidedignidade (82,1\%). Entretanto, é importante ressaltar que foram utilizadas análises adequadas para o processo de validação, como o uso da análise fatorial confirmatória, além dos procedimentos sugeridos para as etapas de teste das qualidades psicométricas deste tipo de escala 9,15.

Por fim, pode-se concluir que a escala apresenta validade, consistência interna e reprodutibilidade adequada para avaliar a autoeficácia para atividade física em idosos brasileiros. 


\section{Resumen}

El presente estudio tuvo como objetivo analizar la validez del análisis factorial confirmatorio y la confianza en la escala de medida de autoeficacia en la actividad física de ancianos. Participaron 118 ancianos (78\% mujeres), cuya edad fue de 60 a 90 años. Para evaluar el análisis factorial confirmatorio se utilizó el programa Mplus 6.1. La confianza fue comprobada por la consistencia interna y la estabilidad temporal. La escala original estaba compuesta por cinco ítems con respuesta dicotómica (no/sí), de manera independiente para la actividad física moderada y vigorosa. El ítem relacionado con la confianza para realizar la actividad física cuando el sujeto está de vacaciones fue excluido. Fueron identificados dos constructos denominados "autoeficacia para caminata" y "autoeficacia para actividad física moderada y vigorosa", con carga factorial $\geq 0,50$. Hubo adecuados valores de consistencia interna, tanto para la caminata $(>0,70)$, como para la actividad física moderada y vigorosa $(>0,80), y$ de estabilidad temporal para todos los ítems. Se concluye que la escala de autoeficacia para actividad física presenta validez, consistencia interna y confianza adecuada para evaluar ese constructo en ancianos brasileños.

Autoeficacia; Estudios de Validación; Actividad

Motora; Anciano

\section{Colaboradores}

R. A. Borges liderou a escrita do artigo, participando de todas as etapas. C. R. Rech contribuiu com as análises e interpretação dos dados, participando também na redação do artigo. S. T. Meurer participou da coleta de dados e colaborou com a redação do artigo. T. R. B. Benedetti coordenou o estudo e revisou o artigo. Todos aprovaram a versão final do manuscrito.

\section{Agradecimentos}

Ao Instituto Lemann pelo apoio financeiro, à Capes pela concessão de bolsa de estudos, aos membros do Grupo de Estudos de Atividades Físicas para Idoso do CDS/ UFSC que executaram a pesquisa e à Prefeitura Municipal de Florianópolis, campo de estudo.

\section{Referências}

1. Bandura A. Self-efficacy: toward a unifying theory of behavioral change. Psychol Rev 1977; 84:191-215.

2. Becofsky K, Baruth M, Wilcox S. Psychosocial mediators of two community-based physical activity programs. Ann Behav Med 2014; 48:125-9.

3. Bandura AA. Social foundations of thought and action: a social cognitive theory. Englewood Cliffs: Prentice Hall; 1986.

4. Wójcicki TR, McAuley E. Manutenção de intervenções de atividade física: premissas para eficácia individual. Revista Família, Ciclos de Vida e Saúde no Contexto Social 2014; 2:182-7.

5. McAuley E, Blissmer B, Katula J, Duncan TE. Exercise environment, self-efficacy, and affective responses to acute exercise in older adults. Psychol Health 2000; 15:341-55.
6. Lee LL, Perng SJ, Ho CC, Hsu HM, Lau SC, Arthur A. A preliminary reliability and validity study of the Chinese version of the self-efficacy for exercise scale for older adults. Int J Nurs Stud 2009; 46:230-8.

7. McAuley E, Jerome GJ, Elavsky S, Marquez DX, Ramsey SN. Predicting long-term maintenance of physical activity in older adults. Prev Med 2003; 37:110-8.

8. Jerome GJ, McAuley E. Enrollment and participation in a pilot walking programme: the role of selfefficacy. J Health Psychol 2013; 18:236-44

9. Bandura A. Guide for constructing self-efficacy scales. In: Pajares F, Urdan TC, editors. Self-efficacy: beliefs of adolescents. Charlotte: Information Age Publishing Inc.; 2006. p. 307-37. 
10. Sallis JF, Pinski RB, Grossman RM, Patterson TL, Nader PR. The development of self-efficacy scales for health-related diet and exercise behaviors. Health Educ Res 1988; 3:283-92.

11. Boff RM. Evidências psicométricas das escalas de autoeficácia para regular hábito alimentar e autoeficácia para regular exercício físico [Dissertação de Mestrado]. Porto Alegre: Pontifícia Universidade Católica do Rio Grande do Sul; 2012.

12. Rech CR, Sarabia TT, Fermino RC, Hallal PC, Reis RS. Propriedades psicométricas de uma escala de autoeficácia para a prática de atividade física em adultos brasileiros. Rev Panam Salud Pública 2011; 29:259-66.

13. World Health Organization. Global recommendations on physical activity for health. Geneva: World Health Organization; 2010.

14. Marôco J. Análise de equações estruturais: fundamentos teóricos, software e aplicações. Lisboa: ReportNumber; 2010.

15. Terwee CB, Bot SD, de Boer MR, van der Windt DA, Knol DL, Dekker J, et al. Quality criteria were proposed for measurement properties of health status questionnaires. J Clin Epidemiol 2007; 60:34-42.

16. Lee L-L, Arthur A, Avis M. Using self-efficacy theory to develop interventions that help older people overcome psychological barriers to physical activity: a discussion paper. Int J Nurs Stud 2008; 45:1690-9.
17. Lee L-L, Chiu Y-Y, Ho C-C, Wu S-C, Watson R. The Chinese version of the Outcome Expectations for Exercise scale: validation study. Int J Nurs Stud 2011; 48:672-80

18. Everett B, Salamonson Y, Davidson PM. Bandura's exercise self-efficacy scale: validation in an Australian cardiac rehabilitation setting. Int J Nurs Stud 2009; 46:824-9.

19. Lee L-L, Avis M, Arthur A. The role of self-efficacy in older people's decisions to initiate and maintain regular walking as exercise: findings from a qualitative study. Prev Med 2007; 45:62-5.

20. Cox KL, Flicker L, Almeida OP, Xiao J, Greenop KR, Hendriks J, et al. The FABS trial: a randomised control trial of the effects of a 6-month physical activity intervention on adherence and longterm physical activity and self-efficacy in older adults with memory complaints. Prev Med 2013; 57:824-30.

21. Malta DC, Moura EC, Castro AM, Cruz DK, Morais Neto OL, Monteiro CA. Padrão de atividade física em adultos brasileiros: resultados de um inquérito por entrevistas telefônicas, 2006. Epidemiol Serv Saúde 2009; 18:7-16.

Recebido em 25/Mai/2014

Versão final reapresentada em 09/Dez/2014

Aprovado em 09/Jan/2015 